\title{
A high gain RFID tag antenna design method of eliminating the interference of mobile communication terminal
}

\author{
Ouyang Guojun ${ }^{1, \text { a }}$ \\ ${ }^{1}$ Guangdong AIB Polytechnic College Department of Computer, Guangzhou 510507 China \\ agzoygj@163.com
}

Keywords: RFID tag; tag antenna; mobile communication interference; anti-interference; UHF band; high gain

\begin{abstract}
China's UHF RFID band of $840 \sim 845 \mathrm{MHz}$ and $920 \sim 925 \mathrm{MHz}$, distance of mobile communication CDMA800, GSM band near, vulnerable to interference in mobile communication system, resulting in demodulation bit error rate increased RFID tags. The interference of mobile communication system formation mechanism analysis, this paper puts forward a design method of UHF mobile communication terminal RFID tag antenna anti interference, deduced the calculation formula of the thickness $\mathrm{H}$ of dielectric substrate, and then use HFSS repeated experiments, to determine the dielectric substrate reasonable relative dielectric constant $\varepsilon_{r}$ value, loss tangent $\tan \delta$ value, which can design a high gain RFID tag antenna anti interference of mobile communication frequency band. The simulation results show that the, the RFID band return loss values S11 were less than $-10 \mathrm{~dB}$, the CDMA800 and GSM band return loss values S11 were greater than -10dB, the RFID band Voltage VSWR values were less than 2, the the CDMA800 and GSM band Voltage VSWR values were greater than 2 . The RFID tag antenna maximum gain of $7.28 \mathrm{dBi}$, the maximum read distance theory valuation $17.66 \mathrm{~m}$.
\end{abstract}

\section{Introduction}

UHF RFID tag without external power supply, and has the characteristics of read-write distance can be up to above $10 \mathrm{~m}$, the data communication at rates up to $640 \mathrm{~K}$, one-time can read thousands of tags, the manufacturing cost is low [1]. In 2013 November, the first RFID in China (tag) the national standard "information technology of radio frequency identification of $800 / 900 \mathrm{MHz}$ air interface protocol" (GB/T 29768-2013) release, UHF RFID technology has become the mainstream technology in the field of RFID in China. But in the application of RFID system in the process, there are still low reading rate, read range is small, easy to be disturbed, resulting in the promotion and popularization of difficulties. Has been building good System fault more also, seriously affecting the normal use. The interference of mobile communication system is a problem need to be solved in the application.

China UHF RFID band for the 840-845MHz and 920-925MHz, near CDMA800(down870 $880 \mathrm{MHz}$, upstream825 835MH) and GSM(down930 $\sim 960 \mathrm{MHz}$, upstream885 915MHz),in the UHF RFID label work, vulnerable to interference in mobile communication system, resulting in RFID tags demodulation bit error rate increased. The source of interference is mainly the mobile communication terminal equipment in the workplace [2]. In this paper, a detailed analysis of the formation mechanism of interference, this paper puts forward a design method of UHF RFID tag antenna anti interference of mobile communication terminal.

In the RFID tab design process, use of HFSS repeated experiment, to determine the dielectric substrate reasonable relative dielectric constant $\varepsilon_{r}$ and Loss tangent $\tan \delta_{\text {value, antenna maximum }}$ gain reaches $7.28 \mathrm{dBi}$, the maximum read distance theory valuation $17.66 \mathrm{~m}$. 


\section{Analysis of the formation process of mobile communication terminal interference}

\section{The equivalent circuit model of label}

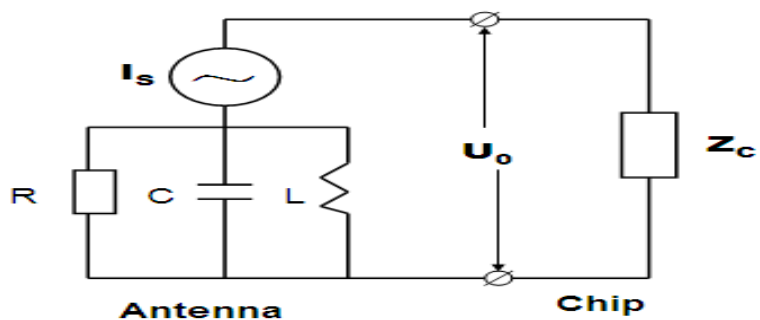

Fig. 1 Equivalent circuit label model

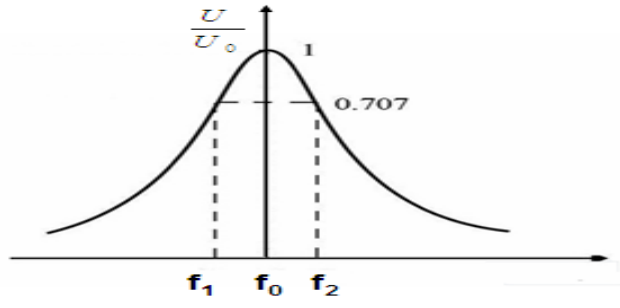

Figure 2 Distribution of the pass band of the antenna circuit

Microstrip antenna is equivalent to a parallel circuit[3], as shown in Figure 1, reader inquiry signal induces a current IS on the antenna input impedance, $\mathrm{ZC}$ chip. The antenna circuit admittance:

$$
Y=G+j(\omega C-1 / \omega L)
$$

The role of $U$ in the end voltage of the chip impedance:

$$
U=\frac{I}{Y}=\frac{I}{1 / R+j(\omega C-1 / \omega L)}
$$

When that happens, parallel resonance, resonant frequency:

$$
f_{0}=\frac{1}{2 \pi \sqrt{L C}}
$$

At this time U maximum value U0. The antenna circuit passband distribution shown in Figure 2, the antenna circuit bandwidth:

$$
B W=f 2-f 1
$$

Said frequency selective antenna, i.e. the signal in this frequency range will likely activate chip, the provisions of China's UHF RFID in the $806 \mathrm{MHz} \sim 960 \mathrm{MHz}$ to the antenna port launch complex shot limit requirements for $-52 \mathrm{dBm}$.

Frequency band mobile communication and RFID China UHF frequency band

Table 1 of China's UHF mobile communication and RFID frequency band

\begin{tabular}{cccccc}
\hline $\begin{array}{c}\text { CDMA800 } \\
\text { Upstream }\end{array}$ & RFID & $\begin{array}{c}\text { CDMA800 } \\
\text { Down }\end{array}$ & $\begin{array}{c}\text { GSM } \\
\text { Upstream }\end{array}$ & RFID & GSM Down \\
\hline $825 \sim 835 \mathrm{MHz}$ & $840 \sim 845 \mathrm{MHz}$ & $870 \sim 880 \mathrm{MHz}$ & $885 \sim 915 \mathrm{MHz}$ & $920 \sim 925 \mathrm{MHz}$ & $930 \sim 960 \mathrm{MHz}$ \\
\hline
\end{tabular}

\section{Interference of mobile communication terminal for RFID Tags}

It is seen from table 1, the CDMA800 uplink frequency band and RFID frequency band 840 $845 \mathrm{MHz}$, GSM uplink and downlink frequency band and RFID frequency band $920 \sim 925 \mathrm{MHz}$ away from the $5 \mathrm{MHz}$, it is easy to produce intermodulation interference and intermodulation interference [4], if the UHF RFID tag antenna frequency selection performance is not ideal, the resonant bandwidth frequency of electromagnetic wave transmitting in mobile communication terminal tag antenna, also be the tag antenna induction and activation of the chip, the mobile communication terminal transmitting electromagnetic wave is coupled to the tag chip, since the information carried by electromagnetic wave is random, coding and signal of UHF RFID system is not the same, leading to tag chip input signal to noise ratio is reduced, RFID Tags Demodulation bit error rate increased [2].

\section{Design method of anti interference of mobile communication terminal}

For convenience of analysis, the table 1 of China's UHF RFID band and UHF band mobile communication range, drawing into UHF frequency classification map, see figure 3. 


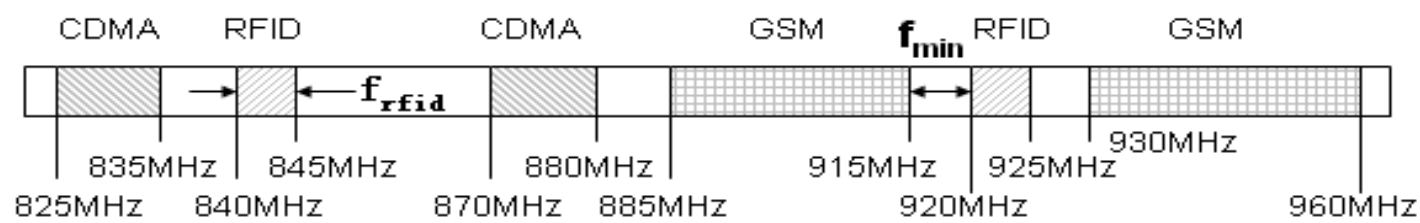

Figure 3 UHF band classification map of China

A RFID band width of $f_{r f i d}$,CDMA, GSM, UHF band from recent distance for RFID band. From Figure 4 we can see, to eliminate the influence of mobile communication frequency band work for the label, the pass band can't antenna covers band part of mobile communication, so the maximum bandwidth of the antenna is:

$$
B W=f_{\text {rfid }}+2 f_{\text {min }}
$$

Because

$$
B W=\frac{(V S W R-1)}{Q_{T} \sqrt{V S W R}}
$$

The quality factor of the total antenna can be expressed as:

$$
\frac{1}{Q_{T}}=\frac{1}{Q_{r}}+\frac{1}{Q_{d}}+\frac{1}{Q_{c}}
$$

Among them, $Q_{r}, Q_{d}, Q_{c}$, the radiation, respectively, dielectric and conductor loss $Q$ value. Due to practical and much larger than the approximate formula, the [5] given:

$$
Q_{T} \cong Q_{r}=\frac{c \sqrt{\varepsilon_{r e}}}{4 f_{r} h}
$$

$\mathrm{C}$ is the speed of light, the effective dielectric constant, the resonant frequency, the thickness of the substrate medium. By (5) to (7) can be obtained:

$$
h=\frac{c\left(f_{r f i d}+2 f_{\min }\right) \sqrt{\varepsilon_{r e}} \sqrt{V S W R}}{4 f_{r}(V S W R-1)}
$$

Reasonable selection of value, loss tangent value, according to (8) type calculation, completed the selection medium substrate material, RFID tag antenna bandwidth not covering CDMA, GSM band, the return loss is a mobile communication system in electromagnetic wave emission antenna, not to trigger chip, antenna has the property of resistance to mobile communication terminal interference good.

\section{Design of a high gain UHF RFID tag antenna}

\section{The tag antenna model structure selection}

This design uses the coax fed microstrip patch antenna structure as prototype design.

The basic structure of tag antenna consists of three parts: the radiation patch, a medium substrate, coaxial cable, as shown in figure 4 .

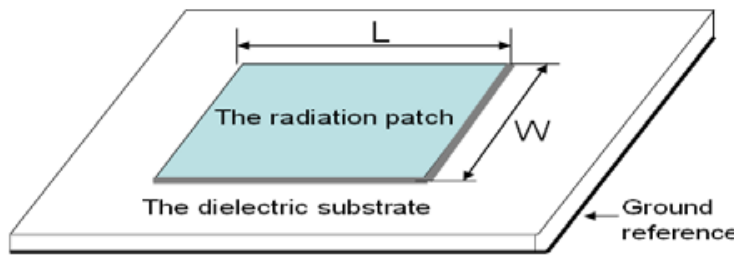

(a) Antenna structure

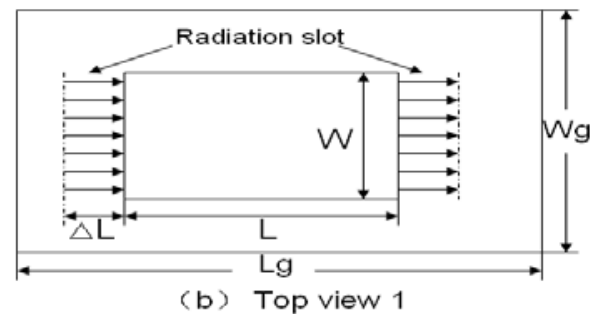

(b) Top view 1 


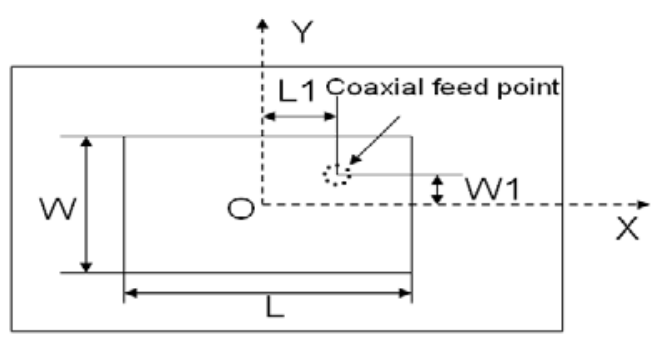

(c) Top view 2

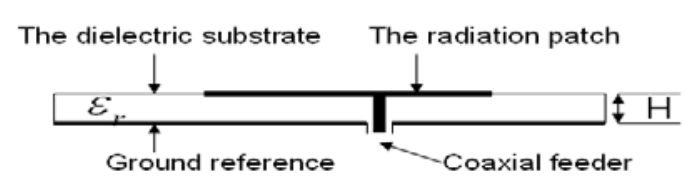

(d) Side view

Figure 4 tag antenna structure

\section{The tag antenna design index}

RFID tag antenna is designed in this paper to follow the "information technology RFID Air Interface Protocol" (GB/T 29768-2013) standard, the design indices:

1) The work center frequency of $842.5 \mathrm{MHz}$ and $922.5 \mathrm{MHz}$.

2) Anti interference performance of tag antenna system: the reflection coefficient $S_{11} \leq-20 d B(V S W R \leq 2.0)$, reflection coefficient of different system $S_{11} \geq-10 d B(V S W R \leq 2.0)$.

3) Maximum gain $G_{a n t} \geq 6 d B$.

4) The theoretical maximum distance $r_{\max } \geq 10 \mathrm{~m}$.

\section{The calculation of radiation patch parameters}

$$
W=\frac{c}{2 f_{r}}\left(\frac{\varepsilon_{r}+1}{2}\right)^{-1 / 2}
$$

Sur $\varepsilon_{r}$ e, h, W, can calculate the effective dielectric constant $\varepsilon_{r e}$.

$$
\varepsilon_{r e}=\frac{\varepsilon_{r}+1}{2}+\frac{\varepsilon_{r}-1}{2}\left(1+12 \frac{h}{W}\right)^{-1 / 2}
$$

Medium wavelength $\quad \lambda_{g}=\frac{c}{f r \sqrt{\varepsilon_{r e}}}$

The radiation patch length $L$ in theory $\lambda_{g} / 2$, because of the influence of edge field, in the design of the size of the $L$ from the $\lambda_{g} / 2$ minus $2 \Delta L$, so:

$$
\begin{aligned}
& \Delta L=0.412 h \frac{\left(\varepsilon_{r e}+0.3\right)(W / h+0.264)}{\left(\varepsilon_{r e}-0.258\right)(W / h+0.8)} \\
& L=\frac{c}{2 f_{r} \sqrt{\varepsilon_{r e}}}-2 \Delta L=0.5 \lambda_{g}-2 \Delta L
\end{aligned}
$$

\section{Substrate size determination}

Distribution of substrate size on the radiation field has no obvious influence, from the view of reducing weight, the antenna installation area and reduce the cost, Lg, Wg should be as small as possible.

Medium plate length $L_{g}=L+0.2 \lambda_{g}$

Medium plate width $W_{g}=W+0.2 \lambda_{g}$

\section{A coaxial feeder position parameters calculation}

This paper adopts orthogonal method to realize dual band multimode.

For rectangular microstrip radiating patch, the feed point is positioned at the $\mathrm{X}$ axis, can stimulate the $T M_{10}$ mode, this time as the center line of the feed point is positioned at the radiation patch Y direction, will not stimulate the $T M_{0 n}(n=1,3,5, \ldots . .$.$) mode. Similarly, when the feed point is$ located in the $\mathrm{Y}$ axis, can stimulate the $T M_{01}$ mode, $T M_{m 0}(m=1,3,5, \ldots . .$.$) mode is not excited. If the$ feed point is placed in the patch near the diagonal position (see Figure 1 (c)), then this can stimulate 
$T M_{01}$ mode and $T M_{10}$ mode, and can obtain the input impedance of the $50 \Omega$, has realized the antenna with dual frequency work.

Feed position calculation formula is as follows [6]:

$$
L_{1}=L\left(1-1 / \sqrt{\xi_{r e}(L)}\right) / 2
$$

In the formula $\xi_{\text {re }}(L)=\left(\varepsilon_{r}+1\right) / 2+\left(\left(\varepsilon_{r}-1\right) / 2\right)(1+12 h / L)^{-1 / 2}$

$$
W_{1}=W\left(1-1 / \sqrt{\xi_{\text {re }}(W)}\right) / 2
$$

In the formula $\xi_{r e}(W)=\left(\varepsilon_{r}+1\right) / 2+\left(\left(\varepsilon_{r}-1\right) / 2\right)(1+12 h / W)^{-1 / 2}$

\section{Simulation and optimization of tag antenna}

\section{Modeling of initial value}

In order to reduce the influence of the mobile communication terminal, as far as possible from the resonant frequency of CDMA, the GSM band is farther. In the design, the definition of resonant frequency values a big first resonant frequency, the resonant frequency of small value a second resonant frequency, can be seen from Figure 4, the first resonant frequency of the antenna resonant frequency of desirable desirable $922.5 \mathrm{MHz}$, second $842.5 \mathrm{MHz}$, the initial parameter calculation of reference when the resonant frequency of the $f_{r}=922.5 \mathrm{MHz}, f_{r f i d}=5 \mathrm{MHz}, f_{\min }=5 \mathrm{MHz}$. Considering the applications without special restrictions installation weight area or volume of the antenna, but for the antenna gain higher, so take $\varepsilon_{r}=2.45$, PTFE base materials $\tan \delta=0.0018$. The coaxial feed line radius $0.6 \mathrm{~mm}$. Take the data into the formula (5) to (17), get the initial design parameters of the antenna are shown in table 2.

Table 2 design variable definition and Simulation of initial value

\begin{tabular}{ccc}
\hline The parameter name & The variable name & $\begin{array}{c}\text { The simulation of initial } \\
\text { value }\end{array}$ \\
\hline The thickness of the substrate medium & $\mathrm{H}$ & $2.7 \mathrm{~mm}$ \\
Dielectric substrate length & $\mathrm{Lg}$ & $142.8 \mathrm{~mm}$ \\
Width of substrate & $\mathrm{Wg}$ & $165.4 \mathrm{~mm}$ \\
The radiation patch length & $\mathrm{L} 0$ & $101.1 \mathrm{~mm}$ \\
The radiation patch width & $\mathrm{W} 0$ & $123.8 \mathrm{~mm}$ \\
The radius of the coaxial feed line & Rfeed & $0.6 \mathrm{~mm}$ \\
The coaxial feed points to the X axis distance & L1 & $17.2 \mathrm{~mm}$ \\
The coaxial feed points to the Y axis distance & W1 & $21.7 \mathrm{~mm}$ \\
1/4 operation wavelength & Length & $81 \mathrm{~mm}$ \\
The radiation boundary length & AirBox_L & L0+2×Length \\
The radiation boundary width & AirBox_W & W0+2 $\times$ Length \\
Radiation boundary height & AirBox_H & $20 \mathrm{~mm}+\mathrm{H}+$ Length \\
\hline
\end{tabular}

\section{Calculation and optimization of performance parameters}

According to the antenna structure parameters obtained, using HFSS modeling and parameter calculation. See the return loss curve of the antenna is shown in Figure 5, the first resonant frequency values for the $916.1 \mathrm{MHz}$, second resonant frequency is $758 \mathrm{MHz}$, we can see that the first resonant frequency value and design index value of the smaller the gap, the second resonant frequency from the design of index value of the larger gap.

According to the previous theoretical analysis, the resonance frequency mode (i.e. first resonant frequency) is mainly determined by the radiation patch in the axial direction length $\mathrm{L} 0$, the resonant frequency of $T M_{10}$ mode (i.e. second resonant frequency) is mainly decided by the radiation patch in the direction of X axis length W0. Show according to (13) type L0 value calculated accurately; (9) type according to the calculated W0 value is big, from the previous analysis, the calculation formula (9) is the maximum value of W0, the application can reduce (according to the repeated experiments, some can be reduced about $10 \%$ ). 


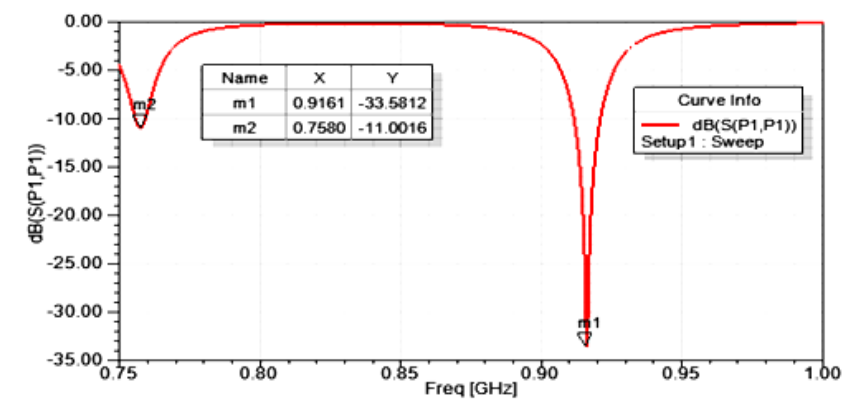

Figure 5 S11 analysis results

$$
\text { ( } \mathrm{L} 0=101.1 \mathrm{~mm}, \mathrm{~W} 0=123.8 \mathrm{~mm})
$$

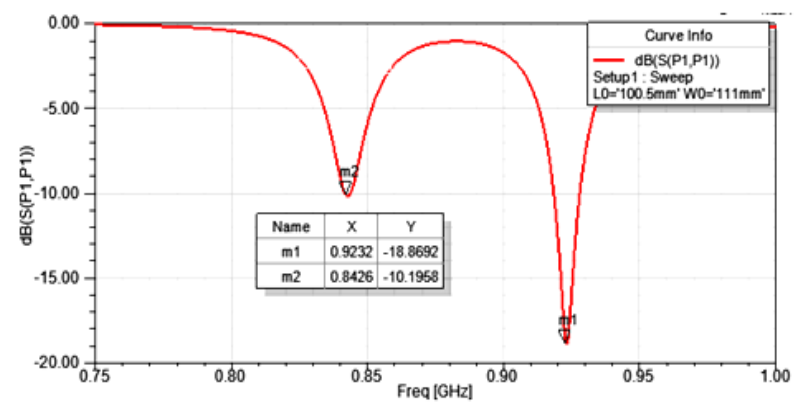

Figure 6 S11 analysis results

(L0=100.5mm, W0 $=111.0 \mathrm{~mm})$

Parameter sweep analysis using HFSS function, respectively adding radiation patch length variable L0 and width of W0 for parameter calculation of scanning, and analyzes the relationship between the antenna resonant frequency and L0, W0. Seen from Figure 6, the L0 $=101.1 \mathrm{~mm}$ first resonance frequency in the vicinity of the $922.5 \mathrm{MHz}$ but less than $922.5 \mathrm{MHz}$, so the range of L0 value is $100 \mathrm{~mm} \sim 101.1 \mathrm{~mm}$, step $0.1 \mathrm{~mm}$ scan analysis, get the $L 0=100.5 \mathrm{~mm}$, a $923.2 \mathrm{MHz}$ value from the $922.5 \mathrm{MHz}$ closest to the. In the scan analysis of W0, according to experience, the first W0 value decreased $10 \%$, namely W0 111.4 as the center, numerical plus or minus $5 \%$ about, namely scanning analysis range $105 \mathrm{~mm} \sim 118 \mathrm{~mm}$, step $2 \mathrm{~mm}$, analysis, narrowing the range, and then analyzes the second scans, the step length of $0.1 \mathrm{~mm}$. Finally, as when $\mathrm{W} 0=111.0 \mathrm{~mm}$, get a $842.6 \mathrm{MHz}$ value from the $842.5 \mathrm{MHz}$ closest to, see Figure 6.

As you can see from Figure 4, after adjustment of L0, W0 values, the resonant frequency achieves the goal of design requirements, but the resonance frequency $923.2 \mathrm{MHz}$ of the S11 value is -18.87 , the resonant frequency of $842.6 \mathrm{MHz}$, the S11 value is -10.20 , not reaching the target requirements.

Adjust the feed position and feed wire core diameter can improve the performance of the antenna [7]. Because of the strong linkage changes feed position and feed wire core diameter on the performance of the antenna, a successively optimization is very difficult to achieve the goal, so need to use the function of HFSS optimal design. The choice of the coaxial feed to the X axis distance L1, the coaxial feed points to the $\mathrm{Y}$ axis distance $\mathrm{W} 1$, coaxial feeder radius Rfeed as optimization design variables, the variation range of L1 is set to $0 \mathrm{~mm} \sim 40 \mathrm{~mm}$, the change range of W1 is set to $0 \mathrm{~mm} \sim$ $45 \mathrm{~mm}$, the change range of Rfeed is set to $0.3 \mathrm{~mm} \sim 0.9 \mathrm{~mm}$, the optimization goal is set to $S_{11}<-20 \mathrm{~dB}$ in $922.5 \mathrm{MHz}, 842.5 \mathrm{MHz}$. The optimization results: L1=16.0mm, W1=16.0mm, Rfeed $=0.6 \mathrm{~mm}$.

\section{The design results}

After optimization, the parameters of the tag antenna is shown in table 3.

Table 3 the value of design variables optimization

\begin{tabular}{ccc}
\hline The parameter name & The variable name & The simulation of initial value \\
\hline The thickness of the substrate medium & $\mathrm{H}$ & $2.7 \mathrm{~mm}$ \\
The radiation patch length & $\mathrm{L} 0$ & $100.5 \mathrm{~mm}$ \\
The radiation patch width & W0 & $111.0 \mathrm{~mm}$ \\
The radius of the coaxial feed line & Rfeed & $0.6 \mathrm{~mm}$ \\
The coaxial feed points to the X axis & $\mathrm{L} 1$ & $16.0 \mathrm{~mm}$ \\
distance & & \\
The coaxial feed points to the Y axis \\
distance
\end{tabular}

\section{Performance analysis of tag antenna}

\section{Anti jamming performance analysis of tag antenna}

The use of HFSS 13 software operation modeling, simulation analysis, tag antenna return loss curve shown in figure 7. 


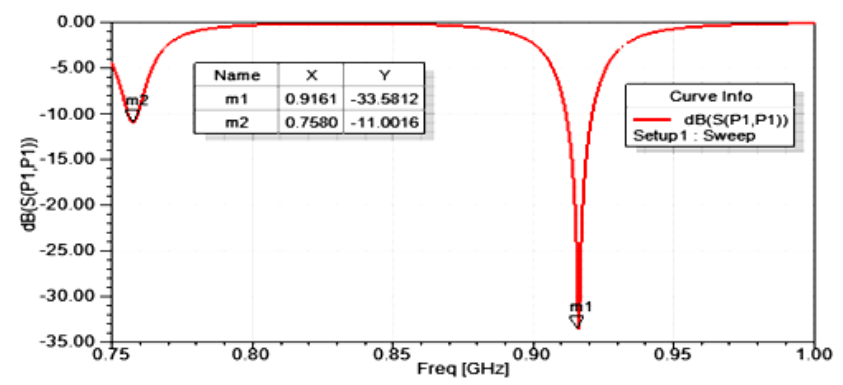

Figure 7 S11 analysis results

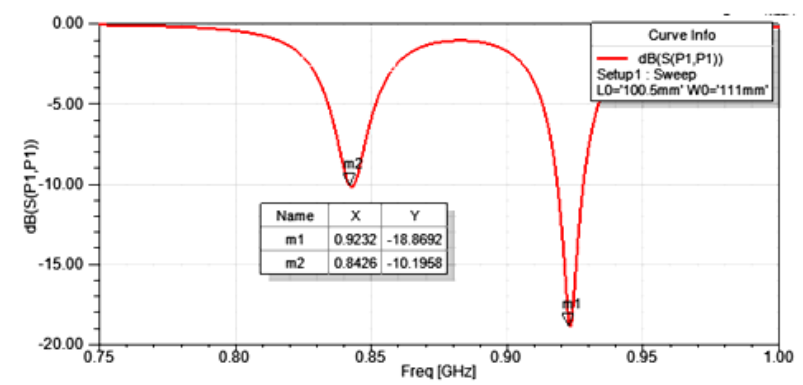

Figure $8 S_{11} \leq-10 d B$ frequency range covers

( $\mathrm{L}=100.5 \mathrm{~mm}, \mathrm{~W}=111.0 \mathrm{~mm}, \mathrm{~L} 1=16.0 \mathrm{~mm}$, $\mathrm{W} 1=16.0 \mathrm{~mm}$, Rfeed $=0.6 \mathrm{~mm})$

From figure 7, the return loss of S11 mobile communication frequency band of the values shown in Table 4, are more than $-10 \mathrm{~dB}$.

Table 4 mobile communication frequency band S11 value

\begin{tabular}{cc}
\hline Band & The value of S11 \\
\hline $825 \sim 835 \mathrm{MHz}$ & $-5.72<\mathbf{S}_{\mathbf{1 1}}<-1.46$ \\
\hline $870 \sim 880 \mathrm{MHz}$ & $-0.90<\mathbf{S}_{\mathbf{1 1}}<-0.76$ \\
$885 \sim 915 \mathrm{MHz}$ & $-5.89<\mathbf{S}_{\mathbf{1 1}}<-0.79$ \\
$930 \sim 960 \mathrm{MHz}$ & $-8.08<\mathbf{S}_{\mathbf{1 1}}<-0.70$ \\
\hline
\end{tabular}

The frequency range of $S_{11} \leq-10 d B$ see Figure $8,840 \mathrm{MHz} \sim 845 \mathrm{MHz}$ frequency band is 838.6MHz $\sim 845.9 \mathrm{MHz}, 920 \mathrm{MHz} \sim 925 \mathrm{MHz}$ frequency range is $919.7 \mathrm{MHz} \sim 928.8 \mathrm{MHz}$, covering all the band of UHF in China RFID.

Tag antenna voltage in Bobbi VSWR as shown in Figure 9, the frequency range of VSWR are shown in Table 5, the frequency of RFID VSWR value of less than 2, the mobile communication frequency band VSWR values are greater than 2.

Table 5 the frequency range of VSWR value

\begin{tabular}{cc}
\hline Band & The value of VSWR \\
\hline $825 \sim 835 \mathrm{MHz}$ & $3.51<$ VSWR $<11.56$ \\
\hline $840 \sim 845 \mathrm{MHz}$ & $1.48<$ VSWR $<1.63$ \\
$870 \sim 880 \mathrm{MHz}$ & $19.04<$ VSWR $<22.79$ \\
$885 \sim 915 \mathrm{MHz}$ & $3.41<$ VSWR $<22.10$ \\
$920 \sim 925 \mathrm{MHz}$ & $1.16<\mathrm{VSWR}<1.78$ \\
$930 \sim 960 \mathrm{MHz}$ & $2.27<\mathrm{VSWR}<23.63$ \\
\hline
\end{tabular}

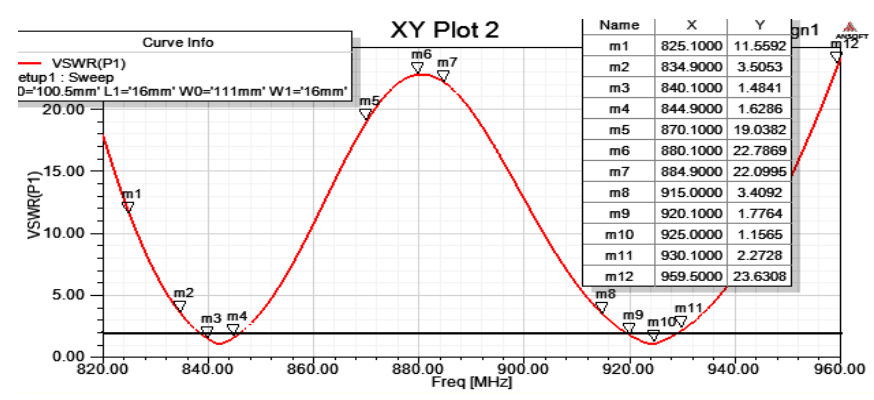

\section{Analysis of the tag antenna gain}

Fig. 9 voltage in Bobbi VSWR analysis results

1. The Smith results and input impedance circle diagram shown in Figure 10, the normalized impedance when $924.2 \mathrm{MHz}$ is $1.0767-\mathrm{j} 0.0342$, namely the antenna input impedance in $924.2 \mathrm{MHz}$ frequency point (53.835-j1.71) $\Omega$, is about $50 \Omega$; normalized impedance $842.4 \mathrm{MHz}$ (1.0602-j0.0832) $\Omega$, which is the input impedance of antenna in $842.4 \mathrm{MHz}$ frequency point for $(53.01-\mathrm{j} 4.16) \Omega$, ca. $50 \Omega$.

2. Three dimensional gain pattern shown in Figure 11, you can see the direction of maximum radiation from the antenna diagram is to the direction method of microstrip patch, the maximum gain value $7.28 \mathrm{~dB}$.

3. The maximum read distance value theory to estimate the label[8]

Assuming the reader and the tag antenna perfect matching and in the same plane, the $\theta_{\text {polarization }}=1.0$ tag antenna polarization loss, Antenna loss $\theta_{\text {antenna }}=0.7$, the reader power $P_{b s E I R P}=4 \mathrm{~W}$, 
reader loss of $\theta_{\text {loadmatching }}$ is 0.8 ; the chip needs minimal reading power $\mathrm{P} 1=35 \mu \mathrm{W}$, label the maximum reading distance of theoretical estimates:

$$
r_{\max }=\sqrt{\frac{P_{b s E I R P} G_{\text {ant }} \lambda^{2}}{(4 \pi)^{2} P_{1}} \theta_{\text {loadmatching }} \theta_{\text {polarization }} \theta_{\text {antenna }}}==17.66(\mathrm{~m})
$$

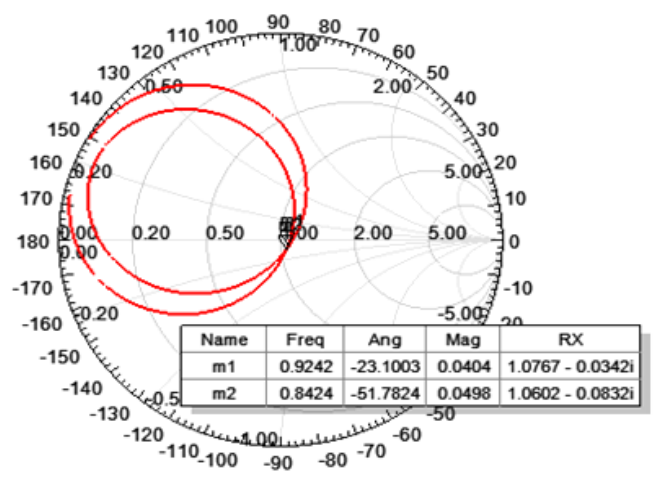

Figure 10 Results S11 Smith chart
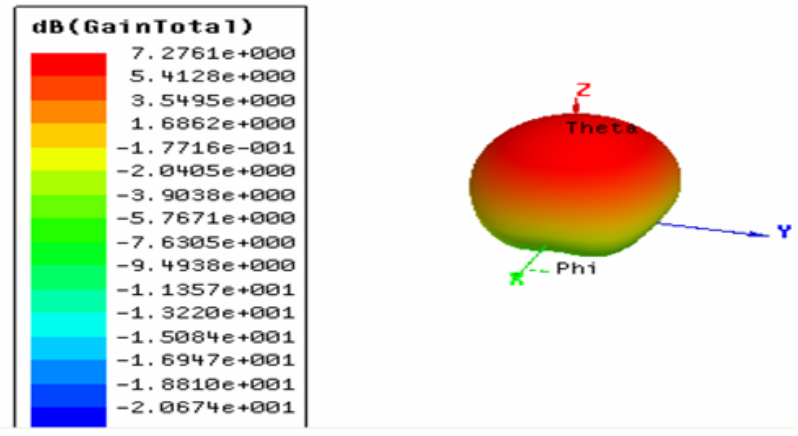

Figure 11 three-dimensional gain pattern

\section{Conclusion}

The simulation in the design of UHF RFID tag antenna in a mobile communication terminal authentication, anti interference design method proposed in this paper are correct, effective. Simulation results show that the calculated thickness, substrate medium according to the $\mathrm{H}$ formula, so to choose the medium substrate as the basis, can guarantee the tag antenna frequency selection performance is good, the ability of anti interference of mobile communication terminal. Because the parameters are chosen properly, the antenna gain is high, the S11 value is small, the input impedance is good, good to meet the design requirements. The design of the antenna structure is simple, easy batch production, and has good application prospect.

\section{References}

[1] Mo Lingfei. Research on UHF RFID anti-metal tag (PhD thesis) [D]. Hangzhou: Zhejiang University, 2009:12.

[2] Shi Dong, Pan Desheng. Interference analysis and suppression strategy of UHF passive RFID system[J]. Telecom Engineering Technics and Standardization, 2012, 6:32-35.

[3] Xia Jijiang, Cao Zhenxin. Effect of the Core Diameter of Feed Coaxial Cable on the Performances of Microstrip Antennas[J] . JOURNAL OF MICROWAVES, 2007,23 (5): 23-25,36.

[4] Shao Huadong, Li Yang, Xue Feng. The Effects of GSM Mobile Phone on the UHF RFID Read Results [J]. ELECTRONICS QUALITY, 2009,12:4-5,16.

[5] Li Yan. Simulation research and design of rectangular microstrip patch antenna(Master degree thesis) [J]. Wuhan: Wuhan University of Technology, 2010:21.

[6] Li Mingyang, Liu Min. HFSS antenna design [M] . Beijing: Publishing House of electronics industry, 2013:125.

[7] Xia Jijiang, Cao Zhenxin. Effect of the Core Diameter of Feed Coaxial Cable on the Performances of Microstrip Antennas[J] . JOURNAL OF MICROWAVES, 2007,23 (5): 23-25,36.

[8] Dominique Paret. The principle and application of ultra high frequency radio frequency identification $[\mathrm{M}]$. Beijing: Publishing House of electronics industry, 2013: 116-145. 